\title{
Weak Logical Constants and Second Order Definability of the Full-Strength Logical Constants
}

\author{
Mitsuhiro OKaDa \\ Université Concordia, Montréal, Canada* \\ and \\ Université de Paris XI, Orsay, France
}

In [5] Prawitz gave a second order definability result on some logical constants, more precisely, he showed that all the intuitionistic logical constants conjunction $(\wedge)$, the first order and the second order existential quantifier $(\exists)$, and disjunction $(\vee)$, are definable on the basis of the intuitionistic implication $(\supset)$, the first order and the second order universal quantifier $(\forall$ ). (Here we should remark that definability of the intuitionistic logical constants implies definability of the classical logical constants via, for example, Gödel's negative interpretation [2]). The use of the full strength intuitionistic implication and universal quantifier as the basis were very essential in Prawitz's method, and one cannot replace those basic constants by the others, e.g. the existential quantifiers and conjunction. In this situation it seems interesting to study a different method of second order definability of the logical constants, especially definability of implication and universal quantifiers, based on the existential quantifiers.

In this note, we remark that if we assume very weak logical constants, the full-strength intuitionistic (hence classical) logical constants, including implication and universal quantifiers, are definable by the help of the intuitionistic second order existential quantifier and conjunction. The weak logical constants introduced here are called "simple" logical constants. As a corollary of our definability result, though the first order system for simple logical constants are extremely weak, the second order extension of that system has the same logical strength as the intuitionistic second order logic (hence as the classical second order logic, via Gödel's negative

* Present address.

This work was partially supported by the Social Sciences and Humanities Research Council (Canada) Grant 410-88-884, Fonds pour la Formation de Chercheurs et l'Aide à la Recherche (Quebec) EQ 4184, Natural Science and Engineering Council (Canada) OGP 36663 and ICR 39976, Committee on Aide to Research Activities (Concordia University) CARA-N42, Ministère de l'enseignment nationale (France).

Annals of the Japan Association for Philosophy of Science, March 1989 
interpratation).

We shall also analyze our simple implication and simple universal quantifier, in terms of Kripke-type possible world models.

\section{§1. Intuitionistic and Simple Logical Constants}

We recall the sequent-style formulation $L J$ of the intuitionistic logic (Gentzen [1], Takeuti [6]).

$$
\begin{array}{cc}
(\rightarrow \wedge) & \frac{\Gamma \rightarrow A \quad \Gamma \rightarrow B}{\Gamma \rightarrow \mathrm{A} \wedge \mathrm{B}} \\
(\wedge \rightarrow) & \frac{A, \Gamma \rightarrow C}{A \wedge B, \Gamma \rightarrow C}, \quad \frac{B, \Gamma \rightarrow C}{A \wedge B, \Gamma \rightarrow C} \\
(\rightarrow \vee) & \frac{\Gamma \rightarrow A}{\Gamma \rightarrow A \vee B}, \quad \frac{\Gamma \rightarrow B}{\Gamma \rightarrow A \vee B} \\
(\vee \rightarrow) & \frac{A, \Gamma \rightarrow C \quad B, \Gamma \rightarrow C}{A \vee B, \Gamma \rightarrow C} \\
(\rightarrow \supset) & \frac{A, \Gamma \rightarrow B}{\Gamma \rightarrow A \supset B} \\
(\supset \rightarrow) & \frac{\Gamma \rightarrow A \quad B, \Gamma \rightarrow C}{A \supset B, \Gamma \rightarrow C} \\
(\rightarrow \forall) & \frac{\Gamma \rightarrow A(a)}{\Gamma \rightarrow \forall x A(x)},
\end{array}
$$

where $a$ is the eigenvariable, i.e., a does not occur in the lower sequent.

$$
\begin{array}{cc}
(\forall \rightarrow) & \frac{A(t), \Gamma \rightarrow B}{\forall x A(x), \Gamma \rightarrow B} \\
(\rightarrow \exists) & \frac{\Gamma \rightarrow A(t)}{\Gamma \rightarrow \exists x A(x)} \\
(\exists \rightarrow) & \frac{(a), \Gamma \rightarrow B}{\exists x A(x), \Gamma \rightarrow B},
\end{array}
$$

where $a$ is the eigenvariable.

Structural Rules :

(Weakening)

(Exchange)

(Cut)

(Contraction)

Axiom :

$$
\underset{\Gamma \rightarrow B}{\stackrel{\Gamma \rightarrow B}{\Gamma} \bar{B}}
$$

$\Gamma, A, B, \Delta \rightarrow C$

$\frac{\Gamma, A, B, \Delta, \Delta \rightarrow C}{\Gamma, B, A}$

$$
\frac{\Gamma \rightarrow A \quad A, \Delta \rightarrow B}{\Gamma, \Delta \rightarrow B}
$$$$
\frac{A, A, \Delta \rightarrow C}{A, \Delta \rightarrow C}
$$

$A \rightarrow A$ 
Second Order Inferences :

$$
(\rightarrow \forall)^{2} \quad \frac{\Gamma \rightarrow A(\alpha)}{\Gamma \rightarrow \forall \varphi A(\varphi)},
$$

where $\alpha$ is the (second order) eigenvariable, i.e., the second order variable $\alpha$ does not occur in the lower sequent.

$$
(\forall \rightarrow)^{2} \quad \frac{A(V), \Gamma \rightarrow B}{\forall \varphi A(\varphi), \Gamma \rightarrow B},
$$

where $V$ is an abstract (cf. [6] $§ 16)$.

$$
(\exists \rightarrow)^{2} \quad \frac{A(\alpha), \Gamma \rightarrow B}{\exists \varphi A(\varphi), \Gamma \rightarrow B},
$$

where $\alpha$ is the eigenvariable.

$$
(\rightarrow \exists)^{2} \quad \frac{\Gamma \rightarrow A(V)}{\Gamma \rightarrow \exists \varphi A(\varphi)},
$$

where $V$ is an abstract.

Next we define simple logical constants $\supset, \vee, \forall, \exists$.

Those simple logical constants are defined in the same way as in the full-strength intuitionistic logical constant in $L J$, i.e., in terms of the left- and right- introduction rules for each logical constant, but the right-introduction rules are restricted to the case in which $\Gamma$ in the above rules is empty, in other words, those are restricted to the following form
(1) $(\rightarrow \supset)$
$\frac{A \rightarrow B}{\rightarrow A \supset B}$
(2) $(\rightarrow \vee)$$$
\frac{\rightarrow A}{\rightarrow A \vee B}, \quad \frac{\rightarrow B}{\rightarrow A \vee B}
$$
(3) $(\rightarrow \forall)$

$$
\underset{\rightarrow \forall F(a)}{\rightarrow \forall x(x)},
$$

where $a$ is the eigenvariable, i.e., a does not occur in the lower sequent.

(4) $(\rightarrow \exists)$

$$
\stackrel{\rightarrow F(t)}{\rightarrow \exists x \bar{F}(x)}
$$

The logical connectives $\supset, \vee, \forall, \exists$ introduced by the above right introduction rules and the corresponding usual left introduction rules are called the simple implication, the simple disjunction, the simple universal quantifier, and the simple existential quantifier, respectively.

Next we consider an extension of the above first order simple logical constants to the second order ones, by restricting the right introduction rules for the second order quantifiers.

(5) $(\rightarrow \forall)^{2}$

$$
\frac{\rightarrow F(\alpha)}{\rightarrow \forall \varphi \tilde{F}(\varphi)}
$$


where $\alpha$ is the eigenvariable, i.e., $\alpha$ is a second order free variable which does not occur in the lower sequent.

(6) $(\rightarrow \exists)^{2}$

$$
\underset{\rightarrow \exists \varphi(V)}{\rightarrow \boxminus(\varphi)}
$$

where $V$ is an abstract.

\section{§2. Second Order Definability}

Now we show that on the basis of the intuitionistic second order existential quantifier and the intuitionistic conjunction, with the help of the simple logical constants, all the (full-strentgh) intuitionistic logical constants are definable.

Now we define the full strength intuitionistic logical constants, as follows.

(1) $A \Rightarrow B \equiv \exists \varphi(\varphi \wedge(A \wedge \varphi \supset B))$

(2) $A V B \equiv \exists \varphi(\varphi \wedge \forall x(\varphi \supset A \vee \varphi \supset B))$

(3) $\forall x F(x) \equiv \exists \varphi(\varphi \wedge \forall x(\varphi \supset F(x)))$

(4) $\exists x F(x) \equiv \exists \varphi(\varphi \wedge \exists x(\varphi \supset F(x)))$

(5) $\forall \psi F(\psi) \equiv \exists \varphi(\varphi \wedge \forall \psi(\varphi \supset F((\psi))$

Then we have the following definability result.

Theorem Each introduction rule for the full strength intuitionistic logical constants is a derived rule, on the basis of the introduction rules for simple logical constants and the intuitionistic rules for $\exists^{2}$ and $\wedge$.

$(\supset \rightarrow)$

$$
\begin{aligned}
& \stackrel{\underline{\alpha \rightarrow \alpha}}{\underline{\alpha, \Gamma \rightarrow \alpha}} \frac{\Gamma \rightarrow A}{\alpha, \Gamma \rightarrow A} \\
& \alpha, \Gamma \rightarrow A \wedge \alpha \quad B, \Delta \rightarrow C \\
& \begin{array}{l}
\frac{A \wedge \alpha \supset B, \Gamma, \alpha, \Delta \rightarrow C}{\alpha \wedge(A \wedge \alpha \supset B), \Gamma, \Delta \rightarrow C} \\
\frac{\exists(\varphi \wedge(A \wedge \varphi \supset B)), \Gamma, \Delta \rightarrow C .}{}
\end{array}
\end{aligned}
$$

$(\rightarrow \supset)$ : Take $C$ as the conjunct $\left(A_{1} \wedge\left(A_{2} \wedge\left(\ldots \wedge A_{n}\right) \ldots\right)\right)$ if $\Gamma$ is of the form $A_{1}, \ldots, A_{n}$.

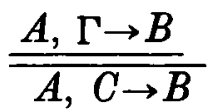

$$
\begin{aligned}
& \overline{\overline{A \wedge C \rightarrow B}} \quad(\rightarrow \supset) \\
& \rightarrow A \wedge C \supset B \\
& \begin{array}{c}
C \rightarrow C \quad C \rightarrow A \wedge C \supset B \\
\hline C \rightarrow C \wedge(A \wedge C \supset B)
\end{array} \\
& \frac{\overline{C \rightarrow \exists \varphi(\varphi \wedge(A \wedge \varphi \supset B))}}{\overline{\Gamma \rightarrow \exists \varphi(\varphi \wedge(A \wedge \varphi \supset B))}}
\end{aligned}
$$

Next we consider $(\rightarrow \forall)^{2},(\forall \rightarrow)^{2},(\rightarrow \vee)$ and $(\vee \rightarrow)$. 
No. 4 Weak Logical Constants and Second Order Definability of the Full-Strength Logical Constants

$(\rightarrow \forall)^{2}$ : Take $C$ as the conjuct of $\Gamma$ as before. Assume that $\alpha$ does not occur in $\Gamma$.

$$
\begin{aligned}
& \frac{\Gamma \rightarrow A(\alpha)}{\underline{C \rightarrow A(\alpha)}} \\
& \rightarrow C \supset A(\alpha) \\
& \frac{C \rightarrow C \rightarrow \forall \psi(C \supset A(\psi))}{C \rightarrow C \wedge \forall \psi(C \supset A(\psi))} \\
& \frac{\overline{C \rightarrow \exists \varphi(\varphi \wedge \forall \psi(\varphi \supset A(\psi))}}{\overline{\Gamma \rightarrow \exists \varphi(\varphi \wedge \forall \psi(\varphi \supset A(\psi))}}
\end{aligned}
$$

$(\forall \rightarrow)^{2}$

$$
\begin{aligned}
& \frac{\alpha \rightarrow \alpha \quad A(V), \Gamma \rightarrow C}{\alpha \supset A(V), \alpha, \Gamma \rightarrow C} \\
& \frac{\frac{\forall \psi(\alpha \supset A(\psi)), \alpha, \Gamma \rightarrow C}{\exists \varphi(\varphi \wedge \forall \psi(\alpha \supset A(\psi)), \Gamma \rightarrow C}}{\overline{\exists \varphi(\alpha)}}
\end{aligned}
$$

$(\rightarrow \vee)$

$$
\frac{\Gamma \rightarrow A}{\frac{\Gamma \rightarrow A}{\rightarrow C \supset A}}
$$$$
\overline{C \rightarrow(C \supset A) \vee(C \supset B)}
$$$$
\Gamma \rightarrow C \wedge((C \supset A) \vee(C \supset B))
$$$$
\Gamma \rightarrow \exists \varphi(\varphi \wedge((\varphi \supset A) \vee(\varphi \supset B)))
$$

$(\vee \rightarrow)$

$$
\frac{\frac{\alpha \rightarrow \alpha A, \Gamma \rightarrow C}{\alpha \supset A, \alpha, \Gamma \rightarrow C} \quad \frac{\alpha \rightarrow \alpha B, \Gamma \rightarrow C}{\alpha \supset B, \alpha, \Gamma \rightarrow C}}{\frac{\alpha \supset A) \vee(\alpha \supset B), \alpha, \alpha, \Gamma, \Gamma \rightarrow C}{(\alpha \supset C)}}
$$

The rules for the full-strength intuitionistic first order $\exists$ and $\forall$ are also derived rules in the similar ways.

In the above proof of definability, we actually used very specific form of the inference rules for the basis logical constants, the second order existential quantifier and the conjunction.

For the second order existential quantifier, we used the following restricted form of $(\exists \rightarrow)^{2}$ rule and $(\rightarrow \exists)^{2}$ rule.

$$
(\exists \rightarrow)^{2} \text {-rule : } \quad \frac{F(\alpha), \Gamma \rightarrow C}{\exists \varphi F(\varphi), \Gamma \rightarrow C}
$$

where $\alpha$ is a propositional variable which does not occur in the lower sequent, and $\varphi$ is a propositional (bound) variable.

$$
(\rightarrow \exists)^{2} \text {-rule : } \quad \frac{\Gamma \rightarrow F(G)}{\Gamma \rightarrow \exists \varphi F(\varphi)},
$$


where $G$ is a subformula of $F$, and $\varphi$ is a propositional (bound) variable.

For the conjunction, we used the usual $(\wedge \rightarrow)$ and the following restricted form of $(\rightarrow \wedge)$.

$$
\frac{A \rightarrow A \quad \Gamma \rightarrow B}{A, \Gamma \rightarrow A \wedge B}
$$

Note that the full-strength intuitionistic second order existential quantifier, $(E)$, and intuitionistic conjunction, \&, are also definable from the restricted forms of rules for $\exists^{2}$ and $\wedge$ above and rules for the simple logical constants extended to the cases for $\exists^{2}$ and $\wedge$. Here new $(\rightarrow \exists)^{2}$ and $(\rightarrow \wedge)$ are of the form

$$
\underset{\rightarrow \exists A(V)}{\rightarrow \psi(\psi)},
$$

where $V$ is an abstract, and

$$
\underset{\rightarrow \mathrm{A}}{\rightarrow \mathrm{A} \wedge \mathrm{B}}
$$

We use the following definitions for the full-strength $E$ and \&.

(6) $(E) \psi F(\psi) \equiv \exists \varphi(\varphi \wedge \exists \psi(\varphi \supset F(\psi)))$

(7) $A \& B \equiv \exists \varphi(\varphi \wedge(\varphi \supset A) \wedge(\varphi \supset B)))$

\section{§3. The Kripke models for the simple logical constants}

In this section we develop a theory of Kripke models for the simple logical constants for $\supset$ and $\forall$. First we give the Kripke models for the intuitionistic propositional logic with the simple implication $\supset$. Then this idea is naturally extended to the Kripke models for the first order logic with the simple logical constants $\supset, \forall$. On the other hand, we use the usual (intuitionistic) rules for $\vee$ and $\exists$, because the simple disjunction and existential quantifier seem too weak to have a reasonable interpretation with Kripke-type semantics : for example, with the simple disjunction $\vee$, the sequent $A \rightarrow A \vee B$ is not provable in general. And with the simple existential quantifier $\exists$, the sequent $A(t) \rightarrow \exists x A(x)$ is not provable in general, either. System $S I$ is obtained from system LJ of the intuitionistic logic by restricting $\supset$ and $\forall$ to the simple logical constants.

We introduce the notion of Kripke model $\langle W, R, \phi\rangle$ as follows. Here $W$ is a non-empty set, where each element $p$ in $W$ stands for (a name of) a possible world. $\leq$ is the accessible relation between possible worlds. $\phi$ is the valuation function such that $\phi: W \times \mathbb{F} \rightarrow\{T, F\} ; \mathbb{F}$ stands for the set of formulas of SI. $\phi(p, A)=$ $T$ is also expressed as $p \models A, \phi(p, A)=F$ as $p \not \neq A$. For the intuitionistic implication, the usual interpretation of $p \models A \supset B$ is "for all $q$ such that $p \leq q, q \| \neq A$ or $q \models$ $B$ " ([3]). On the other hand, for our implication, instead of the condition "for all 
$q$ such that $p \leq q$ ", we only consider a certain subset of the $W$. This subset depends on the formula $A \supset B$. This is denoted by $R(p, \mathrm{~A} \supset \mathrm{B}) . \quad q \in R(p, A \supset B)$ is also denoted by $q R_{A \supset B} p$.

(i) Condition on $p \models$

(1) $P \models A \wedge B$ iff $\mathrm{p} \models$ Aand $p \models B$.

(2) $p \models A \vee B$ iff $p \models A$ or $p \models B$.

(3) $p \models A \supset B$ iff $\forall \mathrm{q} \in \mathrm{R}(p, A \supset B)(q \not \models A$ or $q \models B)$.

(ii) $R_{\mathrm{A}}$ is self-reflexive, i.e., $p \in R(p, A)$, for all $p \in W$ and for all $A \in \mathbb{F}$.

\section{Definition}

(1) $A$ formula $\mathrm{A}$ is true in a model $\langle W, R, \phi\rangle$ iff for all $p \in W, p \models A$.

(2) $A$ formula $\mathrm{A}$ is valid iff $A$ is true in any model $\langle W, R, \phi\rangle$.

The following soundness and completeness theorems hold.

Theorem (Soundness)

If $\rightarrow A$ is provable in $S I$ then $A$ is valid.

Froof

We prove the following proposition by induction on the length of $S I$ proofs. ( $^{*}$ If a sequent $A_{1}, \ldots, A_{n} \rightarrow B$ is provable in $S I$, then for any model $\langle W, R, \phi\rangle$ and for any $p \in W, p \models A_{1}, \ldots, p \models A_{n}$ implies $p \models B$.

Since other cases are trivial, we only consider the case in which the last inference rule is $(\rightarrow \supset)$ of the form

$$
\frac{C \rightarrow D}{\rightarrow C \supset D}
$$

Consider an arbitrary model $\langle W, R, \phi\rangle$. By the induction hypothesis, for any $p$ $\in W, p \models C$ implies $p \models D$. Hence, for any $p \in W$ and for an arbitrarily chosen $R(p, C \supset D)(\subset W), \forall q \in R(p, C \supset D)(q \models C$ implies $q \models D)$. Therefore, $p \models C \supset$ $D$.

Theorem (Completeness)

If a formula $\mathrm{A}$ is valid then $\rightarrow A$ is provale in $S I$. Proof

We define $S I^{\prime}$ which is equivalent to $S I . \quad S I^{\prime}$ is obtained from $L K$ of Gentzen [1] (not $L J)$ by restricting $(\rightarrow \supset)$ rule as before. i.e., $(\rightarrow \supset)$ rule is of the form

$$
\underset{\rightarrow A \supset B}{\rightarrow A} \text {. }
$$

A sequent of $S I^{\prime}$ is of the form, $\Gamma \rightarrow \Delta$, where $\Delta$ as well as $\Gamma$ is an arbitrary finite sequence of formulas. Remark that for any sequent $\Gamma \rightarrow \Delta$ of $S I, \Delta$ consists of at most only one formula. The equivalence of $S I$ and $S I^{\prime}$ can be seen easily from the following Lemma, which is provable by induction on the length of $S I^{\prime}$ proofs.

Lemma

$$
\text { If } S I^{\prime} \vdash \Gamma \rightarrow B_{1}, \ldots, B_{n} \text {, then } S I \vdash \Gamma \rightarrow B_{1} \vee \ldots \vee B_{n} .
$$


The cut elimination theorem for $S I^{\prime}$ is provable by the same reduction procedure in $[1]$.

Then we prove the Theorem in the following stronger form.

Take a condition $\left({ }^{*}\right)$ as follows :

$\left({ }^{*}\right)$ For any model $\langle W, R, \phi\rangle$, and for any $p \in W, p \not \models C_{1} \wedge \ldots \wedge C_{n}$ or $p \models D_{1} \vee \ldots$ $\vee D_{m}$.

Then ( $\left.{ }^{*}\right)$ implies that $C_{1}, \ldots, C_{n} \rightarrow D_{1}, \ldots, D_{m}$ is provable in $S I^{\prime}$.

We first assume that $C_{1}, \ldots, C_{n} \rightarrow D_{1}, \ldots, D_{m}$ is not provable in $S I^{\prime}$. Then we construct a counter model for $C_{1} \wedge \ldots \wedge C_{n} \rightarrow D_{1} \vee \ldots \vee D_{m}$, (by using a cut-free refutation tree).

Starting with $\Gamma^{0} \rightarrow \Delta^{0} \equiv C_{1}, \ldots, C_{n} \rightarrow D_{1}, \ldots, D_{m}$ we continue to follow one of the following cases successively, till none of the cases can be applied.

Assume that $\Gamma^{n} \rightarrow \Delta^{n}$ is already constructed.

(Case 1) if $A \wedge B \in \Delta^{n}$, then $\Gamma^{n+1} \equiv \Gamma^{n}$ and

$$
\Delta^{n+1} \equiv\left\{\begin{array}{l}
\Delta^{n} \cup\{A\}-\{A \wedge B\} \text { if } \Gamma^{n} \rightarrow \Delta^{n} \cup\{A\}-\{A \wedge B\} \text { is not provable } \\
\Delta^{n} \cup\{B\}-\{A \wedge B\} \text { otherwise }
\end{array}\right.
$$

(Case 2) if $A \vee B \in \Delta^{n}$, then $\Gamma^{n+1} \equiv \Gamma^{n}$ and $\Delta^{n+1} \equiv \Delta \cup\{A, B\}-\{A \vee B\}$.

(Case 3) if $A \wedge B \in \Gamma^{n}$, then $\Gamma^{n+1} \equiv \Gamma^{n} \cup\{A, B\}-\{A \wedge B\}$ and $\Delta^{n+1} \equiv \Delta^{n}$.

(Case 4) if $A \vee B \in \Gamma^{n}$, then $\Delta^{n+1} \equiv \Delta^{n}$ and

$$
\Gamma^{n+1} \equiv\left\{\begin{array}{l}
\Gamma^{n} \cup\{A\}-\{A \vee B\} \text { if } \Gamma^{n} \cup\{A\}-\{A \vee B\} \rightarrow \Delta^{n} \text { is not provable } \\
\Gamma^{n} \cup\{B\}-\{A \vee B\}, \text { otherwise }
\end{array}\right.
$$

(Case 5) if $A \supset B \in \Gamma^{n}$, then

(1) $\Gamma^{n+1} \equiv \Gamma-\{A \supset B\}$ and $\Delta^{n+1} \equiv \Delta^{n} \cup\{A\}$, if $\Gamma^{n}-\{A \supset B\} \rightarrow \Delta^{n} \cup\{A\}$ is not provable.

(2) $\Gamma^{n+1} \equiv \Gamma^{n} \cup\{B\}-\{A \supset B\}$ and $\Delta^{n+1} \equiv \Delta^{n}$, otherwise.

Take $\Gamma_{<0\rangle} \equiv \Gamma^{n}, \Delta_{<0\rangle} \equiv \cup \Delta^{n}$. Next enumerate all the elements of $\Delta_{<0\rangle}$ of the form $A \supset B$, say, $A_{1} \supset B_{1}, \ldots, A_{k} \supset B_{k}$. Take $\Gamma \equiv\left\{A_{i}\right\}$ and $\Delta \equiv\left\{B_{i}\right\}$, and repeat the same process as before with $\Gamma \rightarrow \Delta$. Then take $\Gamma_{<0, i>} \equiv \cup \Gamma^{n}$ and $\Delta_{<0, i>}=\cup \Delta^{n}$. Then again enumerate all the elements of $\Delta_{<0, i>}$ of the form $A \supset B$, say, $A_{1} \supset B_{1}, \ldots$, $A_{l} \supset B_{l}$. Take $\Gamma \equiv\left\{A_{j}\right\}$ and $\Delta \equiv\left\{B_{j}\right\}$. Then we have $\Gamma_{\langle 0, i, j\rangle}$ and $\Delta_{<0, i, j\rangle}$. We repeat this procedure again and again untill the procedure stops.

Now we consider the following model $\langle W, G, \phi\rangle: W=\left\{p \mid \Gamma_{p} \rightarrow \Delta_{p}\right.$ appears in this construction procedure .

$G$ is defined so that the following are satisfied: If $A \supset B \in \Gamma_{p}$ then $G(p, A \supset$ $B)=\{p\}$. If $A \supset B \in \Delta_{p}$, then $G(p, A \supset B)=\left\{p, p^{*} 0, p^{*} 1, \ldots, p^{*} k\right\}$; here, when $p$ $\equiv\left\langle i_{0}, \ldots, i_{n}\right\rangle$ then $p^{*} j \equiv\left\langle i_{0}, \ldots, i_{n}, j\right\rangle$ and when $p^{*} e \in W$ then $0 \leq e \leq k$ holds, i.e. $k$ is the number of branchings at the node $p$ minus 1 , in the tree construction above.

Let $P$ be a propositional variable. $\Phi_{\mathrm{G}}(p, P)=T$ if $P \in \Gamma_{p}$. Otherwise $\Phi_{\mathrm{G}}(p$, 
$P)=F$. Then the value of $\Phi_{\mathrm{G}}(p, A)$ for any formula $A$ is determined.

Then we can easily see by induction on the logical complexity of a formula $A$ that if $A \in \Gamma_{p}$ then $\Phi_{\mathrm{G}}(p, A)=T$ and if $A \in \Delta_{p}$ then $\Phi_{\mathrm{G}}(p, A)=F$. In particular, $\Phi_{\mathrm{G}}\left(\langle 0\rangle, C_{i}\right)=T$ for $0 \leq i \leq n$ and $\Phi_{\mathrm{G}}\left(\langle 0\rangle, D_{j}\right)=F$ for $0 \leq j \leq m$. Hence $\Phi_{\mathrm{G}}\left(\langle 0\rangle, C_{i}\right.$, $\left.\wedge \ldots \wedge C_{n} \supset D_{1} \vee \ldots \vee D_{m}\right)=F$. End of Proof.

We extend our completeness result to the first order case with the simple quantifier $\forall$ introduced in section 1. For simplicity of argument, we assume that there is no function symbol in our language. Also without loss of generality, we assume that there is at least one individual constant $c$ in our language.

We can naturally extend the definition of a Kripke model $\langle W, R, \phi\rangle$ to $\langle W$, $D, R, \phi>$ where $D$ is a function such that $D: W \rightarrow \mathbb{T}$, where $\mathbb{T}$ is the set of terms. $D_{p}(\equiv D(p))$ is interpreted as the domain of the world $p$.

We can extend the satisfaction relation $p \models$ to the case of the first order logic in the usual way, here we add the following in the condition on $p \models$.

(4) $p \models \forall x A(x)$ iff $\forall q \in R(p, \forall x A(x)) \forall t \in D_{q}(q \models A[t])$.

(5) $p \models \exists x A(x)$ iff $\exists t \in D_{p}(p \models A[t])$.

The Soundness Theorem is provable in te same way as before. The proof of the completeness Theorem needs the following modification.

For the construction steps of $\Gamma^{n+1} \rightarrow \Delta^{n+1}$, we add the following cases.

(Case 6) if $\forall x A(x) \in \Gamma^{n}$, then $\Delta^{n+1} \equiv \Delta^{n}$ and $\Gamma^{n+1}=\Gamma^{n} \cup\{A(t)\}$, whehe $t$ is a term which already appears in the construction of $\Gamma^{n} \rightarrow \Delta^{n}$, and which did not appear by another application of (Case 6). (If there is no term in $\Gamma^{n} \rightarrow \Delta^{n}$, then we chose constant $c$ ).

(Case 7) if $\exists x A(x) \in \Delta^{n}$, then $\Gamma^{n+1} \equiv \Gamma^{n}$ and $\Delta^{n+1} \equiv \Delta^{n} \cup\{A(t)\}$, where $t$ satisfies the same condition above.

(Case 8) if $\exists x A(x) \in \Gamma^{n}$, then $\Delta^{n+1} \equiv \Delta^{n}$ and $\Gamma^{n+1} \equiv \Gamma^{n} \cup\{A(a)\}$, where a is a free variable which does not appear in $\Gamma^{n} \rightarrow \Delta^{n}$.

Take $\Gamma_{<0\rangle} \equiv \cup \Gamma^{n}, \Delta_{<0\rangle} \equiv \cup \Delta^{n}$.

We next modify the construction process from $\Gamma_{<0>}$ and $\Delta_{<0>}$ to $\Gamma_{<0, i>}$ and $\Delta_{<0, i>}$, as follows.

First enumerate all the elements of $\Delta_{<0>}$ of the form $A \supset B$ or $\forall x A(x)$, say, $A_{0}$ $\supset B_{0}, \forall x A_{1}(x), \ldots, A_{2 n} \supset B_{2 n}, \forall x A_{2 n+1}(x), \ldots$ For $i$, take $\Gamma \equiv\left\{A_{2 i}\right\}$ and $\Delta \equiv$ $\left\{B_{2 i}\right\}$, and repeat the same process as before with this $\Gamma \rightarrow \Delta$. Then take $\Gamma_{<0,2 i>} \equiv$ $\bigcup_{n \in \omega} \Gamma^{n}$ and $\Delta_{<0,2 i>} \equiv \bigcup_{n \in \omega} \Delta^{n}$. For $i$, take $\Gamma \equiv \phi$ (the empfy set) and $\Delta \equiv\left\{A_{2 i+1}(a)\right\}$, where $a$ is a new free variable, i.e. a free variable which did not appear in the former construction process. Then repeat the same process as before with this $\Gamma \rightarrow \Delta$ and take $\Gamma_{<0,2 i+1\rangle} \equiv \bigcup_{n \varepsilon \omega} \Gamma^{n}$ and $\Delta_{<0,2 i+1>} \equiv \bigcup_{n \varepsilon \omega} \Delta^{n}$. This step can be generalized to the step for constructing $\Delta_{<0, i, \ldots, j>}$ and $\Gamma_{<0, i, \ldots, j>}$, as before.

The following condition is added for the definition of $G$ : 
if $\forall x A(x) \in \Gamma_{p}$ then $G(p, \forall x A(x))=\{p\}$. If $\forall x A(x) \in \Delta_{p}$, then $G(p, \forall x A(x))=$ $\left\{p, p^{*} 0, p^{*} 1, \ldots, p^{*} k, \ldots\right\} . \quad D_{p}$ is defined to be the set of terms occurring in $\Gamma_{p}$ or $\Delta_{p}$

\section{References}

1. G. Gentzen, Untersuchungen über das logische Schliessen, Mathematische Zeitschrift, vol. 39 (1935), pp. 176-210, 405-431 ; English translation in his Collected papers, North-Holland, Amsterdam, 1969, ed. M.E. Szabo.

2. K. Gödel, Zur intuitionistischen Arithmetik und Zahlentheorie, Erg. Math. Kolloq. 4, 34$38,1933$.

3. S. Kripke, Semantical analysis of intuitionistic logic, in Formal system and recursive function theory, ed. J.N. Crossley and M.A.E. Dummett, North-Holland, Amsterdam.

4. M. Okada, A thoery of weak implications, Journal of Symbolic Logic 53, No. 1 (1988), 200-211.

5. D. Prawitz, Natural Deduction, Stockholm, 1965.

6. G. Takeuti, Proof Theory, North-Holland, 1987 (2nd edition). 